\title{
NUEVOS VIALES, AFECCIÓN AGRARIA Y COMPENSACIONES TERRITORIALES: POLÍGONOS AGRÍCOLAS Y ENTIDADES DE GESTIÓN DE SUELO RURAL
}

\author{
Juan Cruz Alberdi Collantes \\ Departamento de Geografía, Prehistoria y Arqueología \\ Universidad del País Vasco
}

\section{RESUMEN}

La realización de infraestructuras viarias afecta generalmente a la evolución de aquellas zonas a la que da servicio. Su ejecución, sin embargo, supone en la mayoría de los casos una afección agresiva para el paisaje. En zonas periurbanas, en las que sobrevive una agricultura intensiva y dirigida al mercado próximo, la ejecución de los viales supone en muchos casos la eliminación de unidades y de enclaves agrarios. La ejecución de viales no plantea medidas compensatorias territoriales para hacer frente a la afección si bien, recientemente, esta tendencia parece alterarse con la adecuación de rellenos surgidos tras la ejecución de la obra para uso hortícola.

Palabras clave: carreteras, agricultura, desarrollo agrario, impacto ambiental.

\begin{abstract}
New roads, agricultural impact and land use compensation: agricultural zones and rural land management bodies. The building of new roads usually helps the surrounding areas to develop. However, in most cases, the construction process has an extremely negative impact on the countryside. In rural areas-where intensive agricultural activity supplies nearby markets-roadworks often lead to the elimination of farms and the surrounding land. When a new road is built, no land use compensation system is provided to make up for any losses. Nevertheless, this trend now appears to be changing with the implementation of compensatory measures on land destined for horticultural use after works have finished.
\end{abstract}

Key words: New roads, intensive agriculture, farm development, environmental impact. 
El desarrollo de las comunicaciones se presenta en la actualidad como una necesidad ligada a la promoción económica y a la mejora de la calidad de vida de las zonas beneficiadas. La disponibilidad de distintas opciones de transporte, la reducción temporal de las distancias o la seguridad viaria son razonamientos esgrimidos a la hora de justificar la realización de nuevas y diferentes infraestructuras.

Las indudables mejoras que la ejecución de nuevos viales de comunicación originan contrasta en algunos casos con los efectos negativos que su realización tiene sobre determinados enclaves o actividades, tanto por la propia ejecución de la obra de infraestructura como por los efectos inducidos, entre otros, la capacidad de atraer usos urbanos hacia sus inmediaciones. Entre los principalmente perjudicados destaca su afección sobre los enclaves rurales algunos de los cuales, especialmente en aquellas zonas próximas a núcleos urbanos, se transforman rápidamente en espacios urbanos.

La ejecución de la infraestructura, por otro lado, no plantea la necesidad de proteger determinados territorios por sus posibilidades o características agrarias. Sus actuaciones ambientales se reducen generalmente a minimizar el impacto que provoca la ejecución de la obra sin tener en cuenta otros trazados o variantes alternativas.

La situación que describimos como generalizada se evidencia con claridad en el estudio del territorio de la Comunidad Autónoma Vasca. El carácter urbano-industrial de sus valles atlánticos desvía el trazado de las infraestructuras hacia los escasos enclaves rurales que perviven en este territorio, poniendo en cuestión su conservación.

La importancia social y ambiental del medio rural de los valles atlánticos promueve la necesidad de minimizar el impacto territorial de estas infraestructuras, situación que se va concretando en iniciativas de mejora y de compensación con suelos agrarios. Centramos este trabajo en el tratamiento que se realiza de la actividad agraria en los proyectos de infraestructura y estudios de impacto ambiental, en sus carencias y necesidades de mejora, así como en la descripción de nuevas iniciativas territoriales que se están planteando a la hora de minimizar el impacto de la vía sobre la actividad agraria.

\section{Afección agraria e infraestructura viaria: legislación ambiental y territorial}

La realización de infraestructuras viarias ha de venir precedida de toda una serie de estudios destinados a analizar el impacto ambiental y territorial que su materialización va a tener sobre aquellos espacios que atraviesa. Asimismo, el diseño del vial y los estudios ambientales relacionados han de respetar la normativa de ordenación territorial vigente.

De aquella que afecta al tratamiento y al mantenimiento de los espacios rurales recogemos a continuación la incidencia que los estudios de impacto ambiental y la normativa de ordenación tienen a la hora de proponer una planificación del vial que minimice el impacto sobre la unidad territorial que atraviesa.

\subsection{Estudio de Impacto Ambiental como corrector de los efectos negativos sobre el medio rural}

La directiva 85/337/CEE establece la obligatoriedad de someter los proyectos de obras e infraestructuras a un procedimiento reglado de impacto ambiental, con carácter previo a su aprobación y ejecución.

Los proyectos sujetos a Evaluación de Impacto Ambiental (EIA) son recogidos en los anexos I y II de la mencionada norma. En el Anexo I se establecen 9 tipos de proyectos 
para los que es obligatorio el procedimiento de EIA, y entre ellos está la construcción de autopistas y vías rápidas ${ }^{1}$.

La Ley de Carreteras vigente vincula la construcción de infraestructuras al procedimiento de Evaluación de Impacto Ambiental corroborando con ello las previsiones del R.D.L. 1302/86 sobre EIA y R.D. 1131/88 que lo desarrolla. Este último contiene el reglamento de EIA que establece, para los proyectos de carreteras, autovías, autopistas, etc., la exigencia de incluir en sus proyectos de construcción las medidas correctoras necesarias para eliminar o reducir su efecto sobre el medio. Con ello queda legalmente requerido el tratamiento de los impactos producidos por las carreteras de nuevo trazado.

La transposición al ordenamiento jurídico español de la Directiva 85/337/CEE se concreta en el R.D.L. 1302/1986, de 28 de junio, Evaluación de Impacto Ambiental, y en el reglamento para su aplicación publicado el 30 de septiembre de 1988 (R.D. 1131/1988). El Reglamento de desarrollo 1131/1988 establece unas directrices que con cierta indefinición, concretan las precisiones técnicas de los proyectos sometidos a EIA, y refleja, a su vez, cual debe ser el contenido de los Estudios de Impacto Ambiental ${ }^{2}$. Entre otros aspectos subraya que la Evaluación de Impacto Ambiental debe ser un proceso metodológico destinado a identificar y predecir la magnitud del impacto sobre el medio afectado, tanto en sus elementos como en sus procesos.

El R.D.L. 1302/1986 establece los contenidos mínimos que deben tener los EIA, y que son los siguientes:

- Descripción del proyecto y sus acciones.

- Inventario ambiental

- Identificación y valoración de Impactos.

- Propuesta de medidas protectoras y correctoras ${ }^{3}$.

Aunque no viene explícitamente recogido, se ha de suponer que la evaluación de actuaciones territoriales ha de centrarse más en la definición de los efectos considerados en su conjunto (ambientales, sociales, territoriales, económicos, etc.) dentro de un esquema de evaluación multicriterio, que en la mera descripción y enumeración de aspectos sectoriales, muchos de ellos secundarios e irrelevantes, en que consiste actualmente la Evaluación de

1 En su anexo 2 especifica que obras, instalaciones o actividades han de someterse a la evaluación de impacto ambiental. En lo referente al transporte incluye los siguientes proyectos: Construcción de autopistas, autovías y líneas de ferrocarril de largo recorrido que supongan nuevo trazado, aeropuertos con pistas de despegue y aterrizaje de una longitud mayor o igual a 2100 metros y aeropuertos de uso particular.

2 Con posterioridad, la Unión Europea emite la DIRECTIVA 97/11 CE DEL CONSEJO de 3 de marzo de 1997 por la que se modifica la Directiva 85/337/CEE relativa a la evaluación de las repercusiones de determinados proyectos públicos y privados sobre el medio ambiente. En el 2000 se publicó el Real Decreto Ley 9/2000 de 6 de octubre, de modificación del Real Decreto legislativo 1302/1986, de 28 de junio, de Evaluación de Impacto Ambiental. Pero en la actualidad, todavía no se ha aprobado la Directiva derivada de esta Posición Común, por lo que tampoco ha sido traspuesta al ordenamiento jurídico español. Pese a ello algunas de las comunidades autónomas españolas, se han anticipado a esta situación, incorporando en su legislación medioambiental procedimientos para el sometimiento a EIA del planeamiento urbanístico. Es el caso del País Vasco, Extremadura, Murcia y Castilla-La Mancha, que tienen una ley con alusiones específicas al planeamiento urbanístico, pero que no se reglamenta.

3 Generalmente los estudios de EIA, según BARRAQUETA (2001), contienen al menos las siguientes fases: descripción del proyecto y acciones que de él se deriven, resumen de las alternativas y justificación de la solución adoptada, inventario ambiental y descripción de las interacciones ecológicas claves, identificación y valoración de impactos tanto en la solución propuesta como en las alternativas, establecimiento de medidas correctoras, programa de vigilancia ambiental y documento de síntesis. 
Impacto Ambiental ${ }^{4}$. Sin embargo, la práctica habitual muestra una situación sensiblemente distinta, ya que la mayor parte de los Estudios de Impacto Ambiental son una mera recopilación de información, en gran medida irrelevante.

En la actualidad, la tendencia general es la realización de EIA en sus diferentes procesos de aprobación, pero siempre después de su redacción. Como consecuencia, las evaluaciones de Impacto Ambiental acaban siendo una obligación «piadosa» para justificar la idoneidad de ciertos proyectos y dotar de un mayor protagonismo a los estudios sobre la Vocación del Territorio y sobre su Capacidad de Acogida como marco de referencia para la definición de las actuaciones a materializar.

Esta situación debe reorientarse, haciendo que los criterios ambientales sean simultáneos, o incluso anteriores, al proceso de concepción y elaboración del proyecto. En este caso, no se hace necesaria la realización de EIA, sino la incorporación de criterios ambientales al proceso de redacción del proyecto en cuestión ${ }^{5}$. Pese a que el Reglamento 1131/88 permite que los EIA, puedan elaborarse en cualquier estadio, es obvio que, teniendo en cuenta que la EIA es un instrumento preventivo mas que corrector, la mayor operatividad se conseguiría en la fase de planificación.

Mientras tanto, las medidas ambientales aprobadas serán generalmente puntuales, sesgadas y meramente correctoras de aspectos ligados más a la propia ejecución de la infraestructura viaria que a la vocación y capacidad de acogida del territorio sobre el que se produce el impacto.

\subsection{La flexibilización de las Directrices de Ordenación favorecen la urbanización rural}

La dinámica general de desarrollo ajena a los valores ambientales ha supuesto consecuencias no deseadas (tipologías de viviendas discordantes con el paisaje rural, ocupación de espacios de alto valor agrológico por urbanizaciones de segunda residencia, trazados inadecuados o sin estudios de impacto de infraestructuras de todo tipo, etc.) que tienen su origen en la falta de planeamiento supramunicipal, en una insuficiente protección del medio natural en los planes de rango municipal y en la fuerte demanda de suelo industrial y residencial.

La realización de las Directrices de Ordenación de la Comunidad Autónoma del País Vasco (DOT), documento de referencia obligatoria para toda normativa de ordenación y planificación de rango inferior que se realiza en la CAV, tiene el objetivo de hacer frente a la descoordinación en materia de ordenación y a las consecuencias territoriales derivadas de la misma.

Las Directrices de Ordenación Territorial de la Comunidad Autónoma Vasca, tienen un carácter vinculante y hacen referencia a normas de menor rango. Éstas sugieren que la mayor idoneidad de la Ordenación es alcanzada mediante consensos, a través de planeamientos indicativos y dotados de cierta flexibilidad, y diferir a la normativa de rango

4 De hecho, como señalan MONZÓN et al. (1992), el reglamento 131/88 señala que todo estudio de impacto ambiental debe comprender, al menos, la estimación de los efectos sobre la población humana, la fauna, la flora, la vegetación, el suelo, la geología, el agua, el aire, el clima, el paisaje y la estructura y la función de los ecosistemas presentes en el área previsiblemente afectada... Hay por tanto dos grandes grupos de efectos a estimar: los que afectan al medio natural en sí mismo considerado y los que afectan a las relaciones humanas con el medio.

5 Coincidiendo con la opinión de SERVET y MARTÍNEZ (2001), si la evaluación de impacto ambiental no se aplicara a los procesos de decisión, ésta perdería eficacia ya que daría lugar a una serie de impactos que hubieran podido ser minimizados y solventados con anterioridad. El momento idóneo de incorporar las determinaciones ambientales al planeamiento es el previo a la redacción del plan, ya que en este se pueden seleccionar localizaciones óptimas del territorio para acoger las actividades o usos acordes con su capacidad de acogida. 
inferior, Planes Territoriales Parciales (PTP) y Planes Territoriales sectoriales (PTS), las medidas operativas con mayor nivel de exigibilidad y concreción ${ }^{6}$. Aun así, las DOT establecen algunos criterios de cumplimiento obligatorio por parte del planeamiento más concreto y que afecta directamente a la ordenación de los espacios rurales ${ }^{7}$.

Así, todo el planeamiento de desarrollo (Planes Generales de Ordenación Urbana, Normas Subsidiarias de Planeamiento, Planes Territoriales Parciales, etc.), debe adecuarse a lo establecido en las DOT como Categorías de Ordenación del Suelo clasificado como No Urbanizable, que son:

\section{- Especial Protección \\ - Mejora ambiental \\ - Forestal \\ - Zona Agroganadera y Campiña \\ - Pastos montanos \\ - Sin vocación de uso definida \\ - Protección de Aguas Superficiales}

Con relación a la protección de los usos agrarios, las DOT debe considerar los factores de capacidad agronómica del suelo y su fragilidad ante procesos de deterioro, como criterio de localización de obras e infraestructuras, delimitando los suelos que deben excluirse del desarrollo urbano y económico al comprometer sus valores ${ }^{8}$. Esta protección debe extenderse además a, independientemente de su productividad, aquellos terrenos cuya conservación resulte importante para la viabilidad de los usos agrarios, con criterios acordes con las líneas de desarrollo rural establecidas por el Plan Estratégico Rural Vasco9.

Las DOT se convierten de este modo en un instrumento trascendental para una adecuada preservación de los terrenos agrícolas de gran valor, sobre las que se apoyan otros documentos y normativas recientes en la CAPV(Plan Estratégico Rural Vasco 1997-2000, Ley de Desarrollo Rural y la Ley de Protección del Medio Ambiente) que hacen especial

6 En el marco de la planificación territorial actual todavía es la prospección municipal la que realiza la ordenación efectiva del territorio de la comarca. Sin embargo, a partir de la aprobación de las Directrices de Ordenación Territorial (DOT) en 1997, existen instrumentos de planificación que prevalecen sobre el planeamiento municipal y que eligen la comarca como ámbito de ordenación. Estos son los Planes Territoriales Parciales, la mayoría de ellos actualmente en proceso de aprobación.

7 Con las Directrices de Ordenación Territorial del País Vasco se inicia el largo y complejo proceso establecido en el artículo 10 de la Ley 4/1990, de 31 de mayo, dirigido a conseguir el marco general de referencia para la formulación de los restantes instrumentos de planificación. A partir de la aprobación de este documento, la administración debe establecer los criterios e intentar compaginar las distintas disposiciones para la ordenación territorial en su fase primera, de tal forma que se eviten las actuaciones al margen de estos principios. Esta Ley crea tres herramientas para la ordenación del territorio: Directrices de Ordenación Territorial (DOT), Planes Territoriales Parciales (PTP) y Planes Territoriales Sectoriales (PTS).

8 El objetivo genérico de las DOT es el de garantizar para cada punto del territorio la conservación de sus valores ecológicos, paisajísticos, productivos y científico-culturales. Respecto a la categoría de ordenación del medio físico el objetivo es constituir una zonificación del territorio en sectores homogéneos definidos en función de su capacidad de acogida.

9 Las DOT, asimismo, son el primer documento que plantea categorías de ordenación del suelo clasificado como no urbanizable. El Planeamiento Municipal o el PTP que contemple crecimientos urbanísticos, permitiendo actividades prohibidas en las DOT en las categorías de Mejora Ambiental, Forestal, Agroganadera y Campiña, Pastos Montanos y Protección de Aguas Superficiales debe contener un estudio de alternativas que justifique tanto su interés social como la imposibilidad de ubicación en suelos de menor valor, y su aprobación definitiva precisará de un informe previo y vinculante de la Comisión de Ordenación Territorial del País Vasco. En cualquier caso, en ausencia de PTP y PTS, el Planeamiento Municipal deberá incluir un estudio de su relación con los fundamentos y objetivos de las DOT (1997, p. 128). 
referencia a la protección del suelo agrícola de alto valor y con elevada vocación agraria, destacando la necesidad de su protección.

Pero hay que reflexionar sobre la utilidad de este tipo de normas autonómicas y en especial sobre el grado de vinculación que deben de tener los instrumentos de ordenación territorial. Aunque es un terreno difícil, se debe llegar a un equilibrio en el que respetando el espacio de cada institución territorial implicada se garantice el establecimiento de un sistema capaz y eficaz en la protección del medio ambiente. El que bien por buscar una viabilidad que se traduce en su mera aprobación, bien porque se derive al planeamiento de desarrollo decisiones que se consideran problemáticas y de esta manera se intentan eludir, puede llevar a realizar un documento «muy flexible» pero poco operativo y que impide el logro de los objetivos que tanto en ese documento como en otros la administración territorial competente se está marcando. Se corre el peligro de que la planificación quede solo en un nivel teórico, sin aplicación efectiva.

De hecho, en el largo proceso que ha supuesto la aprobación de las DOT, la Directriz del Medio Físico ha estado cuestionada desde diversos ámbitos. A ello se ha unido la cultura de entender que cualquier planificación que no sea de tipo indicativo o que cuestione el proyecto de cualquier infraestructura supone una imposición y un obstáculo al posible desarrollo económico ${ }^{10}$.

La inercia de la ocupación del territorio sigue adelante, avivada más si cabe por una época de bonanza económica y por el discurso de algunos agentes económicos y sociales que piensan que la continua realización de infraestructuras es la panacea de la solución cuando en muchas ocasiones inducen y agravan los problemas que en teoría querían solucionar viéndose obligados de nuevo a proponer y proyectar nuevas infraestructuras o la ampliación de las actuales. Ante ello, las directrices de obligado cumplimiento se convierten en orientativas y finalmente son ignoradas ante un desarrollo territorial poco ordenado y que atiende casi en exclusividad a intereses urbanos ${ }^{11}$.

\subsection{Tratamiento actual de las tierras agrícolas en la realización de infraestructuras}

Realizando un análisis del ámbito territorial en el País Vasco se confirma que desde el urbanismo y el planeamiento municipal se ha dado un tratamiento disperso y variado del suelo no urbanizable, a veces con regulaciones no adecuadas o contradictorias, y en general con una escasa consideración de los valores naturales y rurales.

El problema para una Administración que busca sobre todo la expansión urbana, es el de conjugar sus objetivos e intereses con la existencia de áreas de reducido impacto ambiental ante la expansión urbana. Pero en este conjunto de objetivos e intereses, la preservación de los ecosistemas de interés a veces ocupa un lugar muy secundario, por lo que la consideración de los efectos ambientales no es tenida en cuenta en absoluto e incluso, como en muchas ocasiones sucede, la variable ambiental no llega a considerarse en la práctica por los urbanistas a la hora de tomar decisiones pese a que el análisis del

10 Sin embargo, al mismo tiempo que se establece esta calificación de usos también se admite la posibilidad de que en función del aval proporcionado por un PTP, un PTS o el Planeamiento General Municipal, un uso calificado como no permitido pase a convertirse en admisible, condicionado a evaluación de la afección sobre la actividad agraria, eso sí, considerando las posibles medidas compensatorias (1999, p. 40).

11 Como se puede apreciar, los criterios de defensa del suelo agrario recogidos en las DOT no se respetan y, a pesar de que se reconoce su importancia para el sector agroganadero e incluso forestal, la ubicación estratégica que presentan corredores, zonas bajas y áreas de reducida pendiente las convierte en espacios de primer orden a la hora de poner en marcha procesos de urbanización. Coinciden mayormente con los escasos fondos de valle que en la comarca quedan sin urbanizar, casualmente las tierras agrarias más productivas. 
medio físico si haya formado parte, normalmente, de los aspectos contemplados en la memoria informativa del proyecto.

La preocupación social por la preservación ambiental se ha asumido formal, pero no realmente por los promotores públicos y privados, lo que hace que los efectos ambientales no tengan una consideración técnica más que aparente.

Muestra de la escasa incidencia que las variables físicas, entre ellas las agrarias, tienen en la configuración final de los proyectos de infraestructuras la recogemos en el tratamiento que de ellas han realizado algunos de los últimos estudios de impacto ambiental que se han realizado en la Comunidad Autónoma Vasca. Tomando como referencia el de la variante del Urumea vamos a observar cómo en proyectos de infraestructura importantes no se hace un estudio de afección agraria ni se tienen en cuenta las prescripciones que las DOT consideran como obligatorias ${ }^{12}$.

El trazado de la autovía transcurre entre zonas relativamente amplias de prados y de riberas en las que las funciones hortícolas son importantes. De entre todas ellas la de Martutene es la más significativa. La huerta intensiva al aire libre domina un amplio espacio en esta zona, completada con superficie destinada a invernaderos y frutales. Los usos forrajeros también tienen presencia, desarrollándose principalmente en las tierras lindates con la ribera. En gran medida se trata de un espacio reliquia en un entorno urbano y montañoso.

Tras analizar la Declaración de Impacto Ambiental del Proyecto de Trazado de la Variante de la carretera GI-131 desde Donostia-San Sebastian a Hernani ${ }^{13}$ se puede corroborar que no hay un estudio que determine el grado de afección de la infraestructura sobre cada una de las explotaciones agrarias de la zona afectada, ni se tiene en cuenta las clases agrológicas ni las categorias de ordenación del PTS Agroforestal y del Medio Natural; se citan pero no se tienen en cuenta. De la lectura de su declaración de impacto ambiental obtenemos las siguientes conclusiones.

- Se reconoce cómo la mayor parte del trazado discurre por suelos laborables y se señala que gran parte del área de estudio pertenece a la categoría de ordenación Agroganadera y Campiña de Alto Valor Estratégico, explícitamente protegida por las DOT y el PTS.

- Sin embargo, se señala que se trata de un tipo de suelo y de unos usos habituales en el entorno, y que por lo tanto no se pierden valores de carácter singular ${ }^{14}$. A pesar de reconocer que parte del trazado discurre en una categoría de ordenación a preservar, se justifican en que las vías de transporte son usos admisibles tras el análisis de alternativas y previa declaración de utilidad pública.

- Respecto a la ocupación del suelo y la pérdida de productividad, se considera la misma como una afección producida en fase de obras, sobre la que no hay posibilidad de establecer medidas correctoras. Por ello, califican el impacto como moderado.

- Reconocen la necesidad de limitar las afecciones al hábitat humano. Con el fin de posibilitar la continuidad de las explotaciones agrícolas afectadas, proponen contar con la colaboración del órgano administrativo competente a efectos de su reubicación.

12 Y ello a pesar de que, como señalan GÓMEZ OREA Y VILLARINO (2000), «las carreteras se suelen manifestar como las infraestructuras más agresivas para el paisaje, consecuencia de una alta rigidez en el trazado y una extensa área afectada por la obra. Recordemos que históricamente, cuando las demandas de tráfico eran reducidas y la construcción estaba menos mecanizada, las carreteras nacionales ocupaban 12 metros de anchura y un radio mínimo que podía ser inferior a 50 metros en zonas de relieve ondulado, mientras en las actuales autovías superan los 40 metros de ocupación con unos radios de giro mínimos de 800 metros».

13 Publicado en el Boletín Oficial de Gipuzkoa del 24-12-02.

14 Como señala GÓMEZ OREA (1992), la realización de infraestructuras en áreas de agricultura extensiva es una actividad compatible sometida a EIA, de ahí la justificación del trazado. Sin embargo, no lo es en aquellos casos en los que se trata de ecosistemas íntegros y paisajes escénicos, y el área afectada bien pudiera tener dicha calificación que ni siquiera es planteado. 
Como se puede observar, en esta Declaración de Impacto Ambiental, a pesar de admitir que la mayor parte del trazado discurre por suelos de gran interés para las explotaciones agropecuarias, no consideran la posibilidad de establecer medidas correctoras justificándose en la consideración del impacto de la infraestructura como moderado ${ }^{15}$.

Por otro lado, con el fin de posibilitar la continuidad de las explotaciones agrícolas afectadas, se apunta a la colaboración del órgano administrativo competente a efectos de su reubicación, sin concretar qué entidad será la encargada de reubicar estas explotaciones, ni de que forma lo harán. Esta afirmación resulta paradójica, especialmente al comprobar que la Comunidad Autónoma Vasca carece de entidad alguna especializada en la gestión de suelo rural o de ejemplo alguno de reubicación por razones agroproductivas ${ }^{16}$.

Queda en evidencia el escaso peso que variables como la productividad del suelo, el concepto de explotación estratégica o justificaciones de tipo paisajístico tienen a la hora de plantearse el diseño de una infraestructura viaria. Los estudios de impacto ambiental son en muchos casos meros planteamientos destinados a justificar la conveniencia de un trazado decidido de antemano.

\section{Aspectos a valorar y mejorar en el estudio y tratamiento de la afección de las actividades agrarias por parte de las infraestructuras}

La realización de una nueva de comunicación acarrea generalmente una pérdida irremediable de suelo agrario. Sin embargo, la consideración de variables de tipo sectorial y el planteamiento de medidas compensatorias a partir de los nuevos espacios que se originan en la ejecución de estas infraestructuras pueden limitar considerablemente las afecciones de este tipo.

\subsection{Recomendaciones para el desarrollo adecuado del procedimiento de Evaluación de Impacto Ambiental}

Para garantizar el éxito de los procedimientos de control ambiental en la ejecución de viales se debe asumir la política de prevención de efectos ambientales que establecen las directivas y políticas europeas, siguiendo procesos secuenciales de planificación en cascada. De esta forma el proceso secuencial debería pasar por cuatro etapas sucesivas:

- En un primer momento, la determinación de los ámbitos sobre los que puede o debe incidir el proyecto, con la definición de sus características fundamentales y en el marco de la planificación territorial. Así los efectos ambientales deben considerarse como restricciones para la selección de ámbitos de actuación allí donde pueden producirse efectos no aceptables.

- Una vez determinado el ámbito del proyecto, debe realizarse un estudio de las variables ambientales del territorio, con la determinación de su capacidad de acogida, detallando las áreas sensibles medioambientalmente con el fin de preservarlas de actividades potenciales poco acordes con su vocación.

15 De hecho, la valoración realizada coincide con una práctica habitual al menos en el País Vasco. Como señala BARRAQUETA (2001), los EIA se elaboran en muchos casos a posteriori, realizando un estudio que se ajusta como un guante a la solución diseñada de antemano, potenciando lo bueno y positivo de la acción incluyendo aspectos absolutamente ajenos al ambiental como es la productividad del suelo o la destrucción de empleo y minimizando e incluso olvidando los aspectos desfavorables.

16 Las soluciones apuntadas distan mucho de lo que deben ser una propuesta de medidas protectores y correctoras. En este caso al menos no se recogen acciones básicas recomendadas en los Estudios de Impacto Ambiental como pueden ser, siguiendo a SERVET Y MARTÍNEZ (2001), la necesidad de describir medidas adecuadas para atenuar o suprimir efectos ambientales negativos o, en su defecto, medidas dirigidas a compensar dichos efectos. La solución de dejar en manos de la administración competente la reubicación de dichas explotaciones cuando la misma no existe es un hecho significativo del carácter de algunos de estos estudios de EIA. 
- El siguiente paso es la selección de alternativas, es decir, adoptada políticamente la decisión sobre la necesidad del proyecto y conocidas las fragilidades del territorio, es necesario particularizar a escala de anteproyecto las posibles alternativas.

- El último nivel a considerar implica la adopción de medidas correctoras de efectos no deseados, tanto directos como externos.

Superado la fase de redacción y durante la fase de ejecución, es imprescindible que se realice un seguimiento detallado de los efectos reales que se van produciendo.

Este esquema secuencial que ha de seguir el procedimiento de EIA de toda obra o infraestructura es aplicable al estudio de unidades territoriales con una clara vocación rural. Respecto a las actividades agrarias al menos se han de evaluar las afecciones, diseñar medidas correctoras y compensar a los afectados garantizando, si ello fuera su deseo, la continuidad de la función agroganadera.

Tomando como referencia los objetivos que persigue el estudio «Evaluación del impacto sobre la actividad agraria de la infraestructura ferroviaria y vasca», realizado bajo la dirección del Departamento de Transportes y del de Agricultua y Pesca de la CAPV, actualmente en proceso de redacción, al menos se deben completar dos fases consecutivas en el estudio de las afecciones sobre la actividad agraria.

a) Evaluación de las afecciones sectoriales:

- Conocimiento de la estructura productiva de las explotaciones y determinación del grado de afección de la infraestructura sobre cada una de ellas.

- Estudio de las infraestructuras agrarias (caminos, regadíos, ...) afectadas por el proyecto.

- Análisis de la afección al entorno rural y natural en su globalidad.

\section{b) Medidas correctoras y compensatorias:}

Creación de un sistema de gestión que facilite las permutas de parcelas entre propietarios y ponga a disposición del sector agrario terrenos marginales, en situación de abandono o próximos al mismo. Con esto se busca:

- Mantener la actividad agraria, minimizando las afecciones sobre el suelo agrario.

- Compensar a los agricultores y ganaderos afectados.

- Corregir la afección por división de la propiedad a ambos lados de la infraestructura.

- Minimizar y corregir la afección a infraestructuras agrarias.

- Crear opciones, allá donde sea posible, de generar disponibilidad de suelo para nuevos agricultores.

La creación de un sistema de compensación no minimizará previsiblemente el impacto paisajístico de la nueva vía sobre un área rural pero garantizará la continuidad de algunas de sus unidades agrarias, básicas para mantener la ruralidad del territorio.

\subsection{Posibilidades de utilización agraria de los nuevos espacios surgidos tras la ejecución de la infraestructura}

El relleno de vaguadas y aprovechamiento de los sobrantes de las infraestructuras es una alternativa a plantear para dar solución a los agricultores que como consecuencia de la construcción de las mismas se quedan sin tierras.

La localización e identificación de los terrenos destinados al depósito de materiales sobrantes debería de constituir requisito del proyecto técnico de la obra de ejecución de la 
infraestructura ${ }^{17}$. Así, su disponibilidad posterior puede quedar operativa tras su declaración de utilidad pública y necesidad de ocupación que venga contemplada en una norma que se elabore al efecto.

La posibilidad de la declaración de utilidad pública se gestiona en los procesos de reversión, que pueden derivarse de la ejecución de la obra de infraestructura, y que dependen de la regulación que del mismo realiza la Ley de Expropiación Forzosa de 16 de diciembre de 1954, modificada parcialmente por la Ley 6/1998 y 38/1999. Según este marco legal, el primitivo dueño puede recobrar la totalidad o la parte sobrante de lo expropiado en el caso de no ejecutarse la obra o no establecerse el servicio que motivó la expropiación, así como si hubiera alguna parte sobrante de los bienes expropiados o desapareciese la afectación, mediante el abono a quien fuera su titular de la indemnización correspondiente.

Las últimas modificaciones, sin embargo, recogen que no habrá derecho de reversión cuando simultáneamente a la desafección del fin que justificó la expropiación se acuerde justificadamente una nueva afectación a otro fin que haya sido declarado de utilidad pública o interés social ${ }^{18}$.

Por otro lado, la Ley 20/1998 de 29 de Junio, de Patrimonio de Suelo que posibilita establecer reservas municipales sobre suelo no urbanizable. Esta ley ha estado vinculada tradicionalmente al logro de terrenos para vivienda de protección oficial, pero hay que destacar que amplia su consideración a otros usos sociales y vincula también el cuadro de medidas al cumplimiento de fines y objetivos contemplados en los instrumentos de ordenación del territorio. En el artículo 21 se apunta la legitimación expropiatoria en la adquisición de terrenos y otros bienes inmuebles, y se dice que la Administración Autonómica y los órganos forales podrán actuar por expropiación, cuando los terrenos y bienes vayan a incorporarse a los patrimonios municipales de suelo o destinarse a la construcción de viviendas de protección oficial, a la promoción pública de suelo para actividades industriales o económicas o a la ejecución de infraestructuras y equipamientos, pudiendo figurar, en su caso, los municipios o demás entidades públicas interesadas como beneficiarios de la expropiación.

La nueva legislación a partir de los años noventa, según Díaz Lema (2001), viene a poner de manifiesto que en lo esencial la propiedad se ha convertido en algo que se puede transformar en dinero, y una vez que se haya asegurado el pago al particular, los avatares sucesivos del objeto expropiado pierden importancia.

La posibilidad de disponer de bolsas de suelo público permitiría crear reservas de terrenos, fundamentados en las posibilidades agrarias del terreno y en arrendamientos a largo plazo dirigidos en primer lugar a aquellos agricultores afectados por procesos de expropiación y, en segundo lugar, a fomentar diversas iniciativas centradas en el desarrollo de la actividad agraria, sin perder por ello el carácter público del suelo utilizado.

17 Siguiendo las aportaciones de GÓMEZ OREA Y VILLARINO (2000), en las infraestructuras gran parte del espacio es externo al directamente ocupado por la infraestructura e ignorado en los proyectos, que centran su atención en los aspectos funcionales, técnicos y sociales. Y sin embargo en muchas ocasiones son indispensables para la integración de la vía, debiendo ser tratados.

18 DÍAZ LEMA (2001), afirma que la ley 6/2001 modifica las normas generales de la reversión en lo relativo a los cambios de uso de las dotaciones públicas, permitiendo en consecuencia a la administración una más amplia libertad de criterio a la hora de utilizar las instalaciones de dominio público. La única exigencia de la ley a este respecto es que ante todo se trate de un uso dotacional público, tanto en el caso antiguo como en el nuevo, y que esté adecuadamente justificado. 


\section{Materialización de proyectos de desarrollo agrario a partir de la realización de infraestructuras viarias}

Las infraestructuras viarias afectan de manera decisiva al desarrollo de las funciones agrarias en los espacios periurbanos en la medida en que reducen las opciones productivas de las explotaciones que se desarrollan en su proximidad. Esta afección puede ser directa, ocupando suelo utilizable, o indirecta, dirigiendo el desarrollo urbano en torno a las nuevas líneas de comunicación. El impacto en valles y espacios de montaña es si cabe más intenso. El desarrollo urbano y las infraestructuras se planifican aprovechado los escasos espacios llanos de estos corredores que quedan sin ocupar, algunos de los cuales permanecen en espera de ser urbanizados mientras otros dan cobijo a una actividad hortícola intensiva y dirigida al mercado que se desarrolla en la proximidad.

La presión social y mediática de estas explotaciones por asegurar su pervivencia está llevando a que se comiencen a plantear soluciones a la falta de suelo, concretándose en iniciativas sustentadas en la intervención que en la ejecución de las vías realizan las instituciones públicas. Recogemos a continuación las acciones principales que en este momento se están barajando en la Comunidad Autónoma del País Vasco.

\subsection{Reutilización de los espacios creados en la realización de infraestructuras: el polígo- no hortícola de Astigarraga}

En la realización de infraestructuras viarias los materiales inertes obtenidos en la excavación generalmente son reutilizados en el reacondicionamiento de la base sobre la que se sustenta el vial. En aquellos casos en los que la excavación es más intensa se adecuan áreas adyacentes, generalmente pequeñas vaguadas, que son rellenadas con los sobrantes de tierras generados. El resultado suele ser la consecución de unos espacios relativamente llanos a cuenta de colmatar pequeñas vaguadas que quedaban interrumpidas por el propio transcurrir de la infraestructura ${ }^{19}$.

La entidad pública responsable expropia los terrenos adyacentes abonando al propietario en función de la utilidad y calificación que presente la parcela. La cantidad aportada habitualmente es poco importante debido a que en la mayoría de los casos se trata de terrenos baldíos. Una vez finalizada la obra estos espacios quedan sin utilidad y el propietario puede solicitar la reversión de su anterior parcela, teniendo para ello que abonar la cantidad económica que la entidad pública había aportado en el momento de la expropiación.

Los espacios creados, de escasa pendiente, llegan a tener en algunos casos una superficie considerable, incluso superior a $5 \mathrm{Ha}$., y con una utilidad en principio limitada a usos no urbanos puesto que el firme resultante no garantiza la plena estabilidad de instalaciones permanentes asentadas sobre ellos. Las funciones agrarias, sin embargo, cuentan de este modo con suelo inerte reconvertible en terreno productivo para uso forrajero y, especialmente, como soporte para el desarrollo de cultivos hortícolas en sustrato inerte o hidroponía.

Las posibilidades de reutilización para uso agrícola de los rellenos resultantes, una herramienta adecuada para compensar con suelo a aquellas explotaciones profesionales expropiadas y cuya continuidad queda irremediablemente condicionada, quedan descarta-

19 El tratamiento de estos espacios, según recogen GÓMEZ OREA Y VILLARINO (2000), se corresponde con zonas donde se depositan materiales de desecho a los que se les suele someter al procedimiento de recuperación general y que se concreta en labores de remodelación y preparación, tal como la eliminación de los elementos gruesos más importantes, refino o escarificado del suelo, hasta obtener una superficie más o menos nivelada y de textura fina, a la que se incorporan las enmiendas si da lugar, para posteriormente aportarles un tratamiento vegetal con fines de estabilización como estética y de integración ambiental. 
das en el momento en el que el propietario solicita la reversión de la parcela, situación que se produce en la práctica totalidad de los casos en los que el terreno ha quedado sensiblemente mejorado. La pérdida de esta opción imposibilita compensar con suelo a un buen número de unidades hortícolas afectadas que se ven abocadas de este modo a desaparecer.

La presión de los horticultores y sindicatos agrarios junto al interés de algunas administraciones por resolver el problema de la carencia de suelo está provocando una reconsideración del derecho de reversión actual y una necesidad de crear suelo público destinado a estos usos. El primero de estos ejemplos se está materializando en el municipio de Astigarraga.

La localidad de Astigarraga se sitúa en el corredor del Urumea a escasa distancia del centro de San Sebastián. Esta ubicación ribereña y próxima a un mercado urbano de entidad ha impulsado el desarrollo de un modelo productivo representado por un caserío de orientación predominantemente hortícola, hasta el punto de hacer de esta zona el área de especialización hortícola principal de la provincia.

La proximidad de San Sebastián y del área industrial que la rodea se materializa en la consecución de corredores urbanos a lo largo de los valles que transcurren por su territorio y que afectan a las zonas de ribera de Astigarraga. La presencia de usos urbano-industriales es manifiesta en toda la vega del bajo Urumea si bien, entre empresas e infraestructuras, determinadas zonas han conservado su fisonomía agraria tradicional.

El relanzamiento urbano-industrial que la comarca está viviendo, tras décadas de recesión económica, ha originado un incremento de la ya tradicional presión urbana sobre el área, a lo que contribuye la pervivencia de espacios sin urbanizar en la zona. Aunque todo tipo de usos urbanos se instalan ahora en esta ribera y en sus proximidades (industriales, servicios, ocio, residenciales...), tal vez sea la incidencia que el diseño de nuevas infraestructuras viarias está teniendo el aspecto más destacado de la presión urbana. De hecho, actuaciones como la nueva circunvalación de la ciudad, el tren de alta velocidad o la autovía del Urumea se proyectan sobre esta zona, aprovechando los «vacíos de oportunidad» que ésta presenta ${ }^{20}$.

De todas ellas, la que mayor impacto va a tener sobre la función agraria de Astigarraga es la realización de la circunvalación o segundo cinturón de San Sebastián cuyo trazado discurre por el cinturón verde que al sur bordea la conurbación San Sebastián-Irun, zona de escasa pendiente y mediana altura con una clara vocación hortícola.

La oposición de los horticultores lleva al consistorio municipal y al Departamento de Obras Públicas y Ordenación del Territorio de la Diputación Foral a plantearse soluciones territoriales para estos agricultores afectados. La importancia del hecho estriba en que es la primera vez que un Departamento de Obras Públicas y Ordenación del Territorio se plantea la necesidad de realizar una compensación que supere la mera indemnización económica. La fórmula propuesta se sustenta en el patrimonio territorial del Ayuntamiento y en las posibilidades que la ejecución de una obra de tales dimensiones supone en la creación de suelo útil para el desarrollo de funciones hortícolas.

Una de las parcelas afectadas por el trazado pertenece al Ayuntamiento de Astigarraga. Con una superficie de alrededor de $30 \mathrm{Ha}$., se corresponde con suelos de pendiente

20 Según el avance del PTP de Donostialdea-Bidasoa, las grandes piezas del medio físico libres de asentamientos urbanos, pero cercanas a éstos y a las redes de comunicaciones son las principales reservas para las futuras posibles implantaciones dotacionales, configurándose como «vacíos de oportunidad». Se debe evaluar cuidadosamente la ordenación de estas áreas con distintas alternativas, su preservación estricta o su especial regulación como zonas de interés agroganadero o forestal, hasta su ocupación urbanística intensiva, pasando por todo tipo de fórmulas intermedias. Lo que no debe hacerse es ignorar las potencialidades de desarrollo urbanístico intensivo que atesoran para el área funcional (1995, p. 132). 
pronunciada, en la mayoría de los casos interfluvios unidos entre sí por medio de collados de reducida extensión. La única superficie (1 Ha.) que presenta posibilidades de ser aprovechada para uso hortícola ha sido cedida a una empresa de jardinería especializada en el trabajo con discapacitados. El resto de la parcela está ocupada por matorral, bosque de ribera y alguno de los collados es aprovechado como pastizal.

El proyecto de la Autopista atraviesa la parcela longitudinalmente por su extremo norte, ocupando el espacio comprendido entre el monte Txoritokieta y el desarrollo de los distintos collados y regatas que cuelgan de él. El desmonte a realizar es importante y la necesidad de rellenar los interfluvios inmediatos la opción más sencilla y efectiva. La actuación permite recuperar una importante superficie para el desarrollo de funciones hortícolas en hidroponía (Ver mapa $n^{\circ} 1$ ).

La superficie de invernaderos media de una explotación profesional, con una media de dos o tres personas empleadas a tiempo exclusivo, tiene en el País Vasco suele rondar entre 0,2 y 0,4 Ha. A horticultura al aire libre suele destinar alrededor de 0,5 Ha. La consecución de una superficie útil próxima a los $10 \mathrm{Ha} \mathrm{m}^{2}$ y destinada a usos hortícolas permitirá que muchas de las explotaciones afectadas, así como otras interesadas en ampliar la superficie utilizable, puedan tener una posibilidad de desarrollo en esta parcela y mantener de este modo una cultura que permanece en estas zonas próximas a la ciudad.

Tan importante como las propias consecuencias del proyecto es el cambio de actitud respecto al problema generado por la falta de suelo que se observa en este caso, así como las posibilidades que surgen cuando distintas administraciones persiguen un fin común. Aunque la pérdida de enclaves rurales en estas áreas es irreparable, con el deterioro de la

MAPA № 1. Circunvalación de San Sebastián y planificación del polígono hortícola de Astigarraga

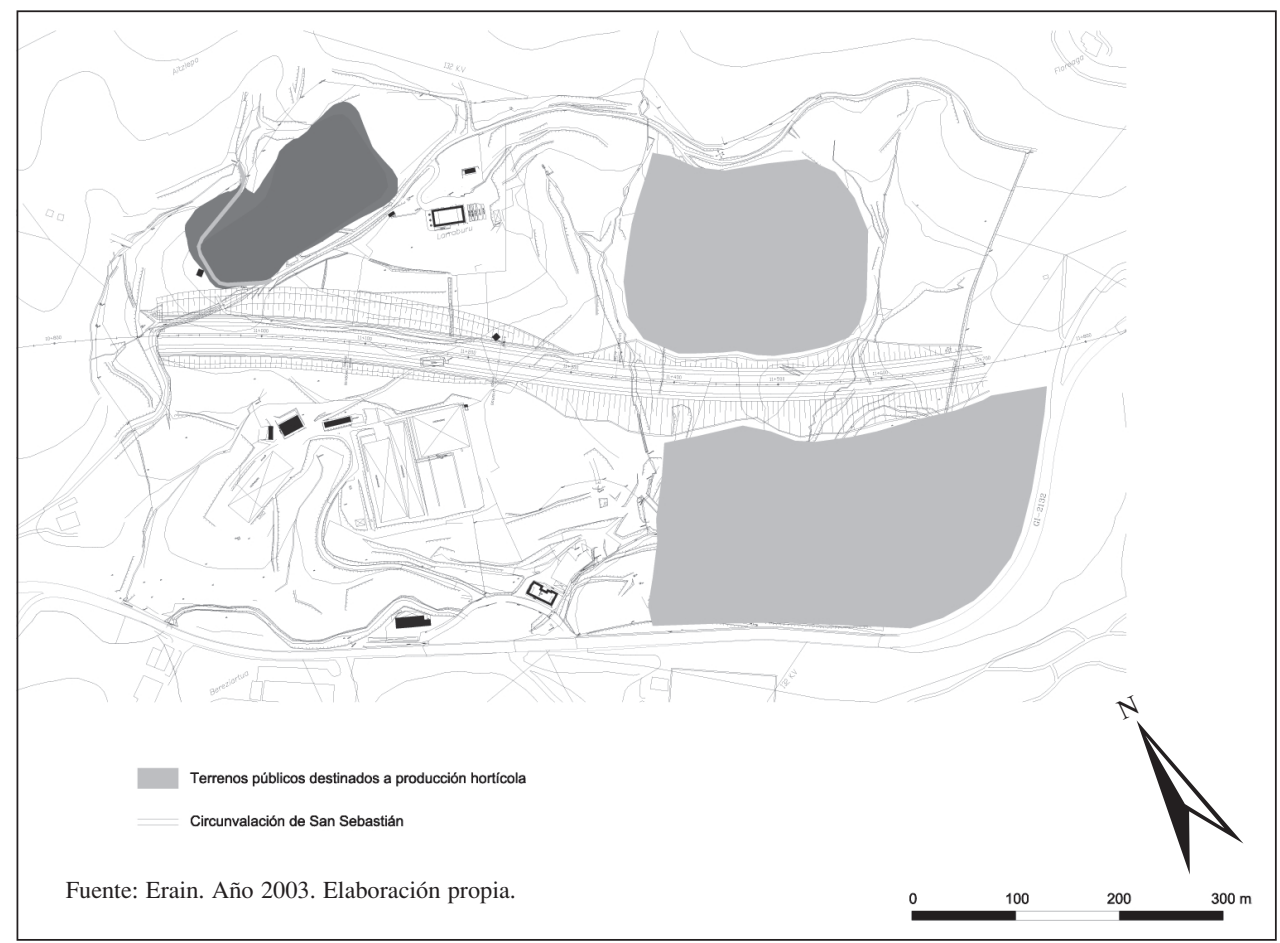


calidad ambiental que ello origina, al menos la perdida de la función productiva sí es remediable.

La acción es, asimismo, un modelo a seguir de manera generalizada en la ejecución de este tipo de infraestructuras, aspecto que va a definir la verdadera prioridad que tiene para las administraciones públicas compensar y desarrollar las funciones agrarias en los territorios más urbanos.

El proyecto del polígono hortícola o «agroaldea» de Astigarraga se concreta en dos fases distintas:

- Una primera fase, actualmente en ejecución, y que no se ve afectada directamente por el trazado del segundo cinturón, si bien éste se desarrolla en su proximidad y condiciona el desarrollo de la parcela. Se trata de un terreno de alrededor de 1,5 Ha., situado en el extremo noroeste de la parcela pública, utilizado para usos forrajeros y con pendientes medias próximas al 10\%. En este caso, se procede a nivelar la parcela mediante excavación hasta aportarle la pendiente adecuada para el desarrollo de las instalaciones que se van a ubicar en la zona, siempre inferior al $4 \%$. Como resultado se obtiene una superficie adecuada para la consecución de dos invernaderos con una superficie media de 0,5 Ha. cada uno (Ver mapa $\mathrm{n}^{\mathrm{o}} 2$ ).

- Una segunda, aún por definir, y cuya ejecución depende de la realización de los rellenos de la autovía. Constituye la reserva de suelo principal con la que compensar a aquellos horticultores que se van a ver afectados por la ejecución del vial. El suelo útil resultante de la operación se aproxima a las 9 Ha.

Otro aspecto a resaltar se fundamenta en la definición de un régimen de funcionamiento del espacio originado. En este caso se apuesta por mantener la propiedad del suelo como pública y que sea el propietario, el ayuntamiento, el que arriende el derecho a disfrute de las parcelas resultantes. Los principios básicos que definen su regulación son los siguientes:

MAPA № 2. Primera fase del Polígono Hortícola de Astigarraga

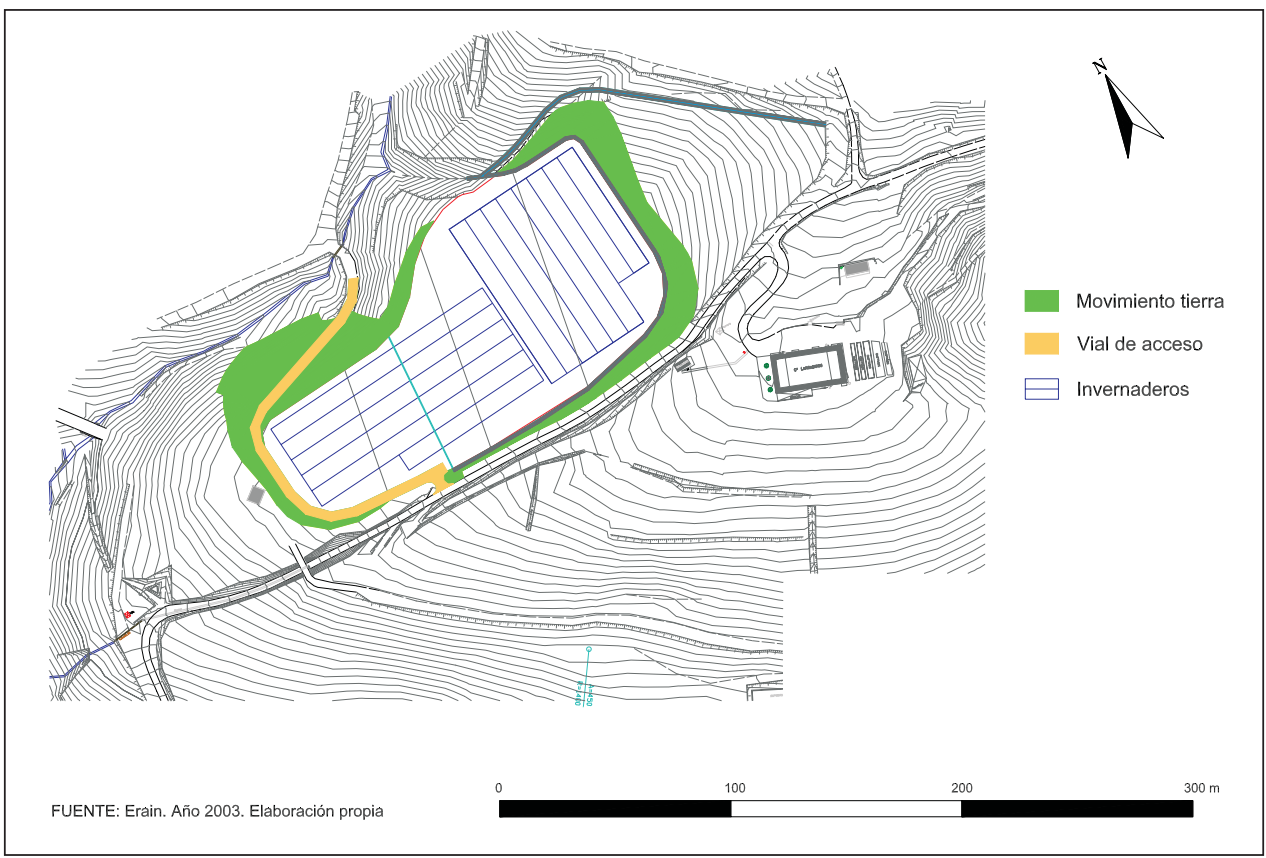


- La cesión se realiza por el máximo de tiempo permitido por la ley, es decir, por un total de 15 años, más 5 años prorrogables y recoge la posibilidad de prolongar el contrato una vez transcurrido el plazo anteriormente estipulado.

- La parcela asignada de cada producto es cedida en régimen de arrendamiento. Se cobra una cantidad anual si bien esta es simbólica (100 Euros.).

- Como todo polígono destinado a una actividad económica, las parcelas cedidas han de estar convenientemente urbanizadas: accesos, explanaciones, agua, electricidad, cierres, ... y esta labor la ha de cumplir la institución responsable del desarrollo rural en la comarca.

- Una vez realizada la cesión corresponde al horticultor instalar su propia explotación. La financiación correrá a cargo del propietario y las líneas de subvención serán las habituales de cualquier proyecto agropecuario, recogidas en el plan de ayudas de las entidades públicas correspondientes.

La falta de terrenos adecuados para el desarrollo de la actividad agrícola en el área atlántica del País Vasco es una realidad contrastada. La incorporación de nuevos agricultores al sector agrario es mínima y entre otras razones la imposibilidad de obtener o arrendar terrenos a un precio adecuado es una de las principales causas. Esta realidad, si cabe, se agrava en el caso del sector hortícola. Aquí, la necesidad de buenos terrenos o al menos con pendientes no muy pronunciadas es todavía mayor. En general, o se tiene terrenos en propiedad o se hace muy difícil incorporar o ampliar explotaciones. En un territorio tan pequeño y con tanta pendiente, este tipo de terrenos se encuentran en valles o vaguadas y en ellas es donde principalmente se asientan los núcleos de población, las actividades económicas y vías de comunicación.

\subsection{Creación de entidades de gestión de suelo rural}

La programación de una gran infraestructura que atraviesa toda la Comunidad Autónoma Vasca, el Tren de Alta Velocidad, línea que comunica todas sus capitales siguiendo un trazado en forma de «Y», genera un impacto territorial con claras repercusiones sobre las funciones agrarias.

La toma de conciencia de la pérdida de suelo productivo que la agricultura va a sufrir lleva a plantearse por parte de las instituciones la creación de una entidad de suelo rural que, aprovechando la envergadura de los movimientos de tierra que la obra a efectuar va a generar, compense con suelo, en la medida de sus posibilidades, a los agricultores afectados. La iniciativa es aprovechada por las instituciones de desarrollo agrario para proponer la creación de una entidad de gestión de suelo rural ${ }^{21}$ que aglutine otras posibilidades de gestión de tierras agrarias, además de las surgidas a partir de la realización del tren de alta velocidad.

El llamado Banco de Suelo se plantea como respuesta a la necesidad de crear una base territorial constituida, en un principio, por todas aquellas explotaciones agrarias afectadas por la vía férrea, para posteriormente poder integrarse por otros bienes inmuebles de naturaleza agraria.

21 La demanda de un órgano público de intervención en el mercado de la tierra agraria es una solicitud ya tradicional de los sindicatos agrarios vascos. Así lo señala el Sindicato EHNE (2002), que apunta como punto de partida la constatación de la existencia de un problema, tierra, que en el ámbito a actual, la CAV, se define como la dificultad o imposibilidad de acceso por parte del productor agrario al factor básico de producción, la tierra, por no existir un mercado de tierra, sino de suelo, y no existir instrumentadas formas de acceso a ese factor de producción distintas al mercado. 
Consecuentemente, los departamentos implicados, Transportes y Obras Públicas, y el de Agricultura y Pesca, representados por el Consejero de Transportes y el Consejero de Agricultura respectivamente, junto a los representantes de los Departamentos de Agricultura de las diferentes Diputaciones Forales organizan el proyecto de Decreto de creación de la Sociedad de Gestión del Banco de Suelo, por medio del cual la Comunidad Autónoma gestiona y administra los bienes inmuebles de naturaleza rústica afectados por la vía férrea del tren de velocidad alta.

En el proyecto se contemplan, entre otros, los siguientes aspectos mínimos:

- La naturaleza jurídica: se trata de un organismo autónomo, dotado de personalidad jurídica, capacidad de obrar y patrimonio propio para el cumplimiento de los fines de la sociedad de gestión del Banco de Suelo 22 .

- Las funciones que ha de desempeñar, de las que destacamos las siguientes:

- La gestión de diferentes formas de adquisición de terreno agrícola, como permutas, ceses anticipados en la actividad agraria, expropiaciones, ejercicio de los derechos de adquisición preferente... ${ }^{23}$

- La gestión de las fincas sobrantes y de propietarios desconocidos, surgidas de la concentración parcelaria. Según la ley 4/1989 publicada en el BOE del 21 de Julio, de ordenación agraria y desarrollo rural, durante el plazo de tres años contados desde la firmeza de la resolución de la concentración, las fincas sobrantes podrán ser utilizadas para la subsanación de los errores que se advierten o como compensaciones. Transcurrido ese plazo se dispondrá de esas tierras, bien para destinarlas a aquellas finalidades que beneficien a la generalidad de los agricultores de la zona o bien para adjudicarlas al municipio.

- La gestión de los sobrantes de terreno expropiado y de los lugares, preferentemente vaguadas, para deposito de materiales sobrantes de excavación. Según el Plan Territorial Sectorial de la red ferroviaria en la CAPV, constituirá requisito del proyecto técnico de la obra la ejecución de la infraestructura ferroviaria, la localización e identificación de los terrenos destinados al deposito de los materiales sobrantes, denominados escombres o vertederos, cuya disponibilidad quedará operada por la declaración de utilidad pública y necesidad de ocupación que venga contemplada en norma de rango legal a elaborar al efecto.

- La gestión de los procesos de reversión que puedan derivarse de la ejecución de la obra de infraestructura, en el marco de la regulación que del mismo realiza la Ley de Expropiación Forzosa de 1954, modificada parcialmente por la Ley de Ordenación de la Edificación 38/1999. A tenor de la modificación el primitivo dueño podrá recobrar la totalidad o la parte del sobrante de lo expropiado en el caso de no establecerse el servicio que motivó la expropiación o bien si desapareciese el motivo de la expropiación, mediante el abono a la entidad expropiatoria de la indemnización correspondiente. Sin embargo, no habrá derecho de reversión cuando simultáneamente a la desafección del fin que justificó la

22 La propuesta de creación de esta entidad de gestión de suelo rural se desarrolla en el Plan Territorial Agroforestal y del Medio Rural de la Comunidad Autónoma del País Vasco, en este momento en revisión. En la misma, respecto a las fórmulas estructurales del órgano de gestión, se plantean dos posibilidades. Por un lado, la creación de un único organismo, formado por las Diputaciones y el propio Gobierno Vasco, o también la posibilidad de que funcione un organismo independiente por cada Territorio Histórico consorciado con el Gobierno Vasco.

23 De hecho, esta es la fórmula principal propuesta por los sindicatos agrarios que operan en la Comunidad Autónoma, la intermediación en operaciones de personas que quieran acogerse al régimen de ayudas por cese anticipado de la actividad agraria (EHNE, 1992), aunque dado el escaso resultado que la medida a tenido en las provincias más urbanas, Gipuzkoa y Bizkaia, la medida se nos antoja insuficiente. 
expropiación se acuerde justificativamente una nueva afección a otro fin que haya sido declarado de utilidad pública o interés social, o cuando la afección al fin que justifico la expropiación se prolongue durante diez años desde la terminación de la obra de establecimiento del servicio ${ }^{24}$.

Las dos primeras fórmulas presentan un origen claramente agrario pero son de difícil aplicación en territorios urbanos como Gipuzkoa o Bizkaia ${ }^{25}$. Iniciativas que tratan de retener suelo a partir de la jubilación anticipada, como en el ejemplo asturiano, o que se fundamentan en los sobrantes de las concentraciones parcelarias, cuando en estos territorios apenas ha habido iniciativa alguna de este tipo, no van a aportar suelo que pueda ser utilizado en la configuración de esta bolsa ${ }^{26}$.

La solución planteada, origen por otro lado del órgano de gestión diseñado, se fundamenta en la utilización de los nuevos espacios surgidos a raíz de la realización de rellenos y escombreras. Su recuperación se plantea en la consideración como bien social de la finalidad que posteriormente va a ser instaurada sobre el mismo, la agrícola, que se asentará sobre un suelo que no va a perder su consideración de utilidad pública.

La creación del órgano de gestión de suelo es, al menos por el momento, un proyecto aún por consolidar. Sin embargo, el carácter de la iniciativa y su reflejo explícito en el Plan Sectorial Agroforestal y del Medio Natural son una muestra de la preocupación social e institucional existente sobre la necesidad de apoyar la continuidad de las funciones agrícolas.

\subsection{Los estudios de impacto ambiental proponen soluciones espaciales al problema de la expropiación agraria}

La necesidad de crear suelo agrario útil a partir de la realización de viales de comunicación comienza a hacerse efectiva no sólo en iniciativas aisladas o documentos de marcado carácter agrario sino también en estudios de impacto ambiental de viales de comunicación rodada recientes, como es el caso de la circunvalación de San Sebastián, proyecto presentado a exposición pública en marzo de 2003.

El $2^{\circ}$ Cinturón de Donostia-San Sebastian transcurre por el área oriental de la conurbación Donostia-Renteria, atravesando en su recorrido el área de campina ubicada entre el Parque Natural de Peñas de Aia y el área urbana de la comarca. Su trazado afecta a algunas de las explotaciones más importantes de la provincia así como al cinturón rural que la bordea, un espacio que combinaba funciones agrícolas y recreativas. Supone además la decisión de trasladar los usos urbanos, el crecimiento de la conurbación, hacia una zona en la que las actividades agrícolas habían progresado de una manera destacada, con una mayor especialización que otras áreas de Gipuzkoa.

24 El PTS propone gestionar un patrimonio compuesto por unos bienes inmuebles formados por el nuevo suelo generado con los depósitos de sobrantes de excavación generados por obras de infraestructura, fincas sobrantes y de propietarios desconocidos de la concentración parcelaria, suelo ocioso de las administraciones locales y fincas cedidas voluntariamente por sus propietarios para ser gestionadas por la entidad, especialmente de agricultores acogidos al plan de jubilaciones.

25 Sería muy difícil poner en marcha en todo el País Vasco-Atlántico iniciativas similares a las que se recogen en la Ley 4/1989 del Principado de Asturias, por medio de la cual se crea el Banco de Tierras. Tan sólo el disponer de una base patrimonial pública lo suficientemente amplia permitiría disponer de un patrimonio mínimo. El precio de la tierra es excesivamente elevado y la presión tan importante que difícilmente se puede acceder a otro tipo de terrenos.

26 Se ha de reconocer, sin embargo, que el origen de la idea de crear bolsas o bancos de suelo hay que situarlo en el régimen sobre cese anticipado tras la reforma de la PAC de 1992. El reglamento CEE 2079/92 previó la creación de servicios y redes privadas pero con autorización pública para organizar las transmisiones de tierras. Igualmente, se establecía que los estados miembros podían asimismo crear un organismo que se hiciera cargo de las tierras cedidas y se comprometiera a cederlas a personas que satisfagan las condiciones recogidas en el reglamento. 
El carácter rural de la zona y la presión ejercida por parte de los agentes agrarios lleva a que por primera vez en el País Vasco un proyecto de infraestructura de carreteras considere como severo el impacto que supone la obra en el medio rural y establece medidas para compensar estos impactos. Hasta este momento, los EIA, como hemos señalado en el ejemplo de la variante del Urumea, obviaban la afección agraria de la infraestructura y no establecían ningún tipo de medidas compensatorias.

Estudiando el proyecto de trazado del $2^{\circ}$ Cinturón y el Estudio de Impacto Ambiental llevado a cabo, se aprecia la toma de conciencia de la pérdida de suelo productivo que la agricultura de la zona va a sufrir, planteándose medidas compensatorias (generación de terrenos cultivables) y soluciones alternativas, aprovechando la envergadura de los movimientos de tierra que la obra va a generar.

Analizando los resultados obtenidos en el inventario ambiental llevado a cabo en el Estudio de Impacto Ambiental, se aprecia que han valorado como severas las siguientes afecciones:

- Alteración de las formas de relieve, tanto por movimientos de tierras asociados al trazado como por la creación de vertederos.

- La ocupación de suelos de elevada capacidad productiva y, en especial, de determinadas explotaciones agropecuarias tradicionales, dedicadas a cultivos forrajeros, pastos, manzanas de sidra y cultivos hortícolas, entre otros. La superficie total de afección a suelos cartografiados como de «alta capacidad» se ha evaluado en unas 26 Has y la ocupación de explotaciones incluidas en los Registros de Explotaciones Agrarias en 35,2 Has.

- Los aspectos relacionados con la pérdida de bienes y rentas se centran principalmente en afecciones derivadas de la necesidad de expropiación de terrenos y edificaciones que, dadas las características del territorio, son mayoritariamente de carácter agrario. Se ha realizado un trabajo de análisis y comparación con los listados de expropiaciones y planos parcelarios, con fichas de Registros de Explotaciones Agrarias.

Entre las medidas compensatorias se proponen actuaciones encaminadas a compensar determinadas afecciones cuya corrección no es posible y que, en este caso, se han centrado en propuestas relacionadas con la ocupación de determinadas explotaciones agropecuarias de elevado valor económico y tradicional.

Las medidas compensatorias definidas han consistido en la selección de emplazamientos y diseño de los vertederos de tierras, de modo que se generen superficies aptas para el uso agropecuario, especialmente desde el punto de vista morfológico. Para ello gestionan los excedentes de tierras generados por la infraestructura y realizan una propuesta de vertederos en todo el recorrido.

De este modo, del conjunto de las 14 zonas de vertido detectadas, en 12 de ellas se han definido plataformas de pendiente moderada que permiten dicho tipo de usos. En su momento se definirá el procedimiento administrativo (reversión, ocupación, cesión, permuta, etc.) que permita su uso por parte de particulares o entidades.

El conjunto de superficies generadas en los vertederos susceptibles de orientarse para su aprovechamiento agropecuario es de 21,5 Ha. repartidas en los municipios de Usurbil, Hernani, Donostia, Astigarraga y Renteria. Se toma como ejemplo el planteamiento del polígono hortícola de Astigarraga y se decide ampliarlo a otros rellenos que se van a efectuar en el conjunto de la obra.

Este Estudio de Impacto Ambiental es acorde con las recomendaciones que establecen las directivas y políticas europeas, y denota un cambio importante en lo que se había hecho hasta ahora en los EIA (mera recopilación de información, descripción y enumeración de aspectos sectoriales). 
El que este EIA considere que la construcción de la infraestructura afecta a terrenos de alta capacidad y explotaciones de elevado valor económico y tradicional y establezca la necesidad de crear medidas compensatorias para evitar en lo posible poner en peligro el futuro de estas explotaciones, supone un paso adelante y abre el camino para otras infraestructuras y proyectos urbanísticos que puedan afectar al suelo agrario.

\section{Hacia la consecución de nuevos espacios productivos: a modo de conclusión}

La agricultura se está convirtiendo en una actividad concentrada en un reducido número de explotaciones mientras la gran mayoría ha optado ya por marginalizar y abandonar progresivamente esta actividad. Por lo general, la superficie cultivable que unos abandonan repercute en el incremento de la de aquéllos que continúan, consiguiéndose de este modo una tipología de explotación con una base territorial que permite obtener una mayor rentabilidad.

La agricultura desarrollada en espacios en los que el precio del suelo no depende de su capacidad agrológica sino de las posibilidades y expectativas urbanísticas que existan sobre él, tiene dificultades para desarrollar unidades productivas territorialmente significativas. El agricultor, una vez abandonada la actividad, no arrienda ni vende su heredad a precios adecuados a las posibilidades económicas de otros que sí continúan. Por lo general conserva la propiedad hasta obtener de ella unos réditos acordes con las expectativas urbanas generadas. De este modo, las explotaciones de los espacios periurbanos tratan de compensar su carencia de suelo utilizable con un modelo productivo más intensivo y con unas producciones adecuadas a la demanda del mercado desarrollado en la proximidad, entre las que destacan las especialidades hortícolas.

La dificultad de encontrar suelo utilizable a unos precios adecuados a sus posibilidades agrológicas en los espacios periurbanos se incrementa con el problema generado como consecuencia del avance del espacio urbanizado, progreso que se realiza a expensas de ocupar suelos agrícolas, normalmente aquellos de menores pendientes y que mejores condiciones agrológicas presentan. Las explotaciones afectadas no pueden compensar la pérdida de terreno y en muchos casos con la expropiación finaliza la actividad de la unidad agraria.

El proceso de avance de los usos urbanos suele estar relacionado con la dotación viaria preexistente o en diseño en la zona. La creación de un nuevo vial incrementa las posibilidad de comunicación de las áreas que lo circundan, que progresivamente se van viendo colmatadas con nuevos desarrollos urbanos. La ciudad tiende a crecer hacia estos nuevos espacios de comunicación.

Salvo determinados enclaves que son valorados por sus características naturales, el avance urbano no reconoce otras funciones en el medio rural e incorpora progresivamente estos espacios. Del mismo modo, el diseño de las infraestructuras viarias se realiza sin tener en cuenta aspectos propios de la naturaleza de las explotaciones agrarias tales como la capacidad productiva de los suelos, su estructura parcelaria, comunicaciones, etc.

Las compensaciones realizadas como consecuencia del proceso de expropiación muestran el escaso interés de los ejecutores por el mantenimiento de las funciones agrarias. La renumeración es exclusivamente económica sin plantearse otro tipo de posibilidades adecuadas a los intereses del agricultor.

El agricultor, por lo general, apenas ha mostrado resistencia al avance de los usos urbanos, incluso en aquellos casos en los que la expropiación generaba la imposibilidad de continuar con la actividad. El nuevo progreso que la ciudad está experimentando en los últimos años, y la consecuente pérdida de suelo útil, está generado en esta ocasión una mayor respuesta por parte de aquellas explotaciones que han fundamentado en la unidad 
familiar su proyecto de futuro y que está provocando un replanteamiento del tratamiento que las infraestructuras han aportado a las actividades y usos agrarios.

La búsqueda de otro tipo de compensaciones genera soluciones territoriales novedosas, de sencilla ejecución y adecuadas a las demandas realizadas por los agricultores. Entre éstas, destaca la posibilidad que la realización de nuevas infraestructuras tiene para generar suelo agrícola útil y público, utilizando para ello vertederos y escombreras que en su ejecución van progresivamente clausurando. La concentración de este tipo de suelos en una entidad de gestión permite crear la base territorial necesaria para iniciar proyectos de desarrollo agrario como polígonos hortícolas o instalación de jóvenes agricultores, al que incorporar, en un segundo momento, terrenos de otra naturaleza (prejubilaciones, concentraciones, derecho de tanteo y adquisición... ).

En regiones urbanas, como el País Vasco Atlántico, la dinamización del sector hortícola pasa en gran medida por la industrialización de la producción, incrementando la cosecha sin aumentar la superficie utilizada. La génesis de espacios productivos a partir de las nuevas superficies creadas en la ejecución de los viales permite desarrollar el concepto de polígono hortícola o Agroaldea, cuyo significado viene derivado de la variante industrial o Industrialdea, coincidiendo con suelos de naturaleza pública que la administración adecua a nivel de parcela y servicios y que cede en arrendamiento al particular para su disfrute.

\section{Bibliografía}

BARRAQUETA, P. (2001): Planificación Urbanística. Propuesta metodológica y casos prácticos. I Jornadas de estudios de impacto ambiental, Facultad de Filología, Geografía e Historia. Vitoria.

DÍAZ LEMA, J.M. (2001): El derecho de reversión expropiatoria. Revista Galega de la administración pública, $\mathrm{n}^{-} 27$, pp. 61-62.

EHNE (2002): Organismo público de intervención en el factor tierra para el sector agrario. Euskal Herriko Nekazarien Elkartasuna. Lemoa.

ERAIN (2003): Proyecto de acondicionamiento de parcela para implantación de Agroaldea. Diputación Foral de Gipuzkoa, Departamento de Agricultura.

GARCÍA TREBIJANO, J.A. (1998): Expropiación y reversión de los terrenos. Excepciones. Revista

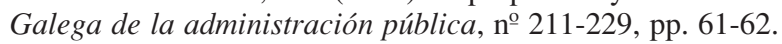

GOBIERNO VASCO. Departamento de Agricultura y Pesca (1992): Plan estratégico rural vasco. Líneas generales de actuación 1992-1996. Vitoria-Gasteiz.

GOBIERNO VASCO. Departamento de Industria Agricultura y Pesca (1996): Plan de actuación para el desarrollo del medio rural vasco 1997-2000. Vitoria-Gasteiz.

GÓMEZ OREA, D. (1992): Planificación rural. Ministerio de Agricultura, Pesca y Alimentación. Editorial Agrícola Española, S.A. Madrid.

GÓMEZ OREA, D. Y VILLARINO (2000): Restauración de espacios afectados por infraestructuras lineales: técnicas para el tratamiento de taludes. Fundación General de la Universidad Politécnica de Madrid. Unidad Didáctica no 9.

MONZÓN, A. y GOMAR, C. (1992): Método de ponderación de la traza para la evaluación de impactos ambientales de carreteras. Estudios Geográficos, nํㅜ 209, pp. 609-636.

OÑORO FERNÁNDEZ, F. -Dir.- (1990): Directrices de Ordenación Territorial de la Comunidad Autónoma del País Vasco. Análisis y Diagnóstico. Gobierno Vasco. Departamento de Urbanismo, Vivienda y Medio Ambiente. Vitoria-Gasteiz.

OÑORO, F. y ZABALA, A. -Dir.- (1995): Avance del P.T.P del Área Funcional de DonostialdeaBajo Bidasoa. Gobierno Vasco. Dept. Urbanismo, Vivienda y Medioambiente. Vitoria-Gasteiz.

SERVET, R. y MARTÍNEZ, N. (2000): Evaluación de impacto ambiental del planeamiento urbanístico: la cuestionabilidad de un procedimiento y una metodología de prevención ambiental. II congreso Internacional de Ordenación del Territorio, pp. 887-902. Gijón.

VEGARA, A. -Dir.- (1997): Directrices de Ordenación Territorial de la Comunidad Autónoma del País Vasco. Gobierno Vasco. Departamento de Ordenación del Territorio, Vivienda y Medio Ambiente Vitoria-Gasteiz. 